\title{
Encrypting Majorana Fermions-qubits as Bound States in the Continuum
}

\author{
L. H. Guessi ${ }^{1,2}$, F. A. Dessotti ${ }^{3}$, Y. Marques $^{3}$, L. S. Ricco ${ }^{3}$, \\ G. M. Pereira ${ }^{1}$, P. Menegasso ${ }^{1,4}$, M. de Souza ${ }^{1}$, and A. C. Seridonio ${ }^{1,3}$ \\ ${ }^{1}$ IGCE, Unesp - Univ Estadual Paulista, Departamento de Física, 13506-900, Rio Claro, SP, Brazil \\ ${ }^{2}$ Instituto de Física de São Carlos, Universidade de São Paulo, C.P. 369, São Carlos, SP, 13560-970, Brazil \\ ${ }^{3}$ Departamento de Física e Química, Unesp - Univ Estadual Paulista, 15385-000, Ilha Solteira, SP, Brazil \\ ${ }^{4}$ Instituto de Física "Gleb Wataghin", Unicamp, Campinas-SP, 13083-859, Brazil
}

\begin{abstract}
We theoretically investigate a topological Kitaev chain connected to a double quantum-dot (QD) setup hybridized with metallic leads. In this system, we observe the emergence of two striking phenomena: i) a decrypted Majorana Fermion (MF)-qubit recorded over a single QD, which is detectable by means of conductance measurements due to the asymmetrical MF-leaked state into the QDs; ii) an encrypted qubit recorded in both QDs when the leakage is symmetrical. In such a regime, we have a cryptographylike manifestation, since the MF-qubit becomes bound states in the continuum, which is not detectable in conductance experiments.
\end{abstract}

PACS numbers: 72.10.Fk 73.63.Kv 74.20.Mn

Introduction.-It is well known that Majorana fermions (MFs) zero-modes[1,2] are expected to appear bounded to the edges of a topological Kitaev chain[3-7]. Interestingly enough, by approaching the Kitaev chain to a quantum dot (QD), the MF state leaks[8] into it and manifests itself as a zero-bias peak (ZBP) in conductance measurements. The latter reveals experimentally the MF-qubit recorded over the QD. Indeed, such a phenomenon was experimentally confirmed in a QD hybridnanowire made by InAs/Al[9] with huge spin-orbit interaction and magnetic fields, being the nanowire placed close to an $s$-wave superconductor. It is worth mentioning that MFs can also emerge in the fractional quantum Hall state with filling-factor $\nu=5 / 2[10]$, in threedimensional topological insulators[11], at the core of superconducting vortices[12-14] and on the edges of ferromagnetic atomic chains covering superconductors with pronounced spin-orbit interaction[15, 16], similarly to semiconducting nanowires[17]. In terms of technological applications, MFs-qubits are of particular interest. This is because of their topological protection against decoherence[3], a key ingredient for the achievement of efficient quantum computers.

In this work, we show that the employment of two QDs, as depicted in Fig.1(a), enables the cryptography of the MF-qubit state $\eta_{\uparrow}=\frac{1}{\sqrt{2}}\left(\Psi_{1}+i \Psi_{2}\right)$ made by the MFs $\Psi_{1}$ and $\Psi_{2}$ with splitting energy $\varepsilon_{M} \rightarrow 0$, where an encoded message can be written over these states of bits. Our main theoretical findings rely on the interplay between the leakage effect and the so-called bound states in the continuum (BICs) $[18,19]$. In this context, it is worth recalling the underlying Physics of such exotic excitations. BICs were proposed by von Neumann and Wigner in 1929[18] as quantum states with localized square-integrable wave functions, but surprisingly within the domain of the energy continuum region. Noteworthy, such states trap particles indefinitely. BICs constitute a current topic of broad interest[20], appearing in several physical systems like graphene[21-23], optics and photonics[24-27], arrangements exhibiting singular
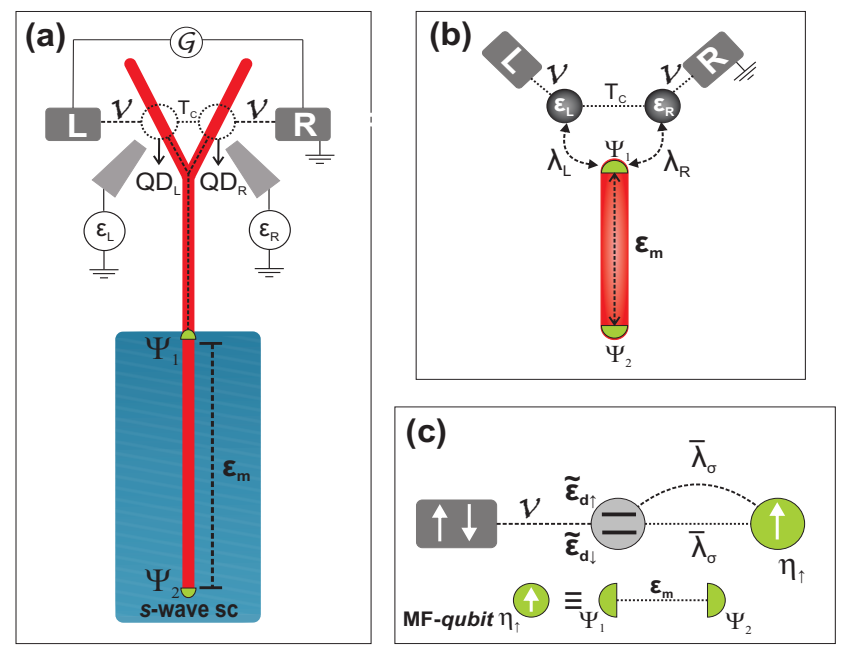

Figure 1. (Color online) (a) Two QDs symmetrically coupled to leads via the hybridization $\mathcal{V}$ and asymmetrically to a topological Kitaev chain by means of the complex amplitudes $\lambda_{L}$ and $\lambda_{R} . \Psi_{1}$ and $\Psi_{2}$ are overlapped MFs with splitting energy $\varepsilon_{M} \rightarrow 0$. (b) Oversimplified sketch of panel (a). (c) Mapping of the original system from panel (b) onto the pseudospin representation. The dressed pseudo-Zeeman gap $\tilde{\epsilon}_{d \uparrow}-\tilde{\epsilon}_{d \downarrow}$ appears depicted within the central QD, which is side-coupled to the qubit $\eta_{\uparrow}$, namely, the MF-qubit. $\bar{\lambda}_{\sigma}$ identifies both the tunneling and the Cooper pair binding energy between the new QD and $\eta_{\uparrow}$, respectively given by the horizontal and semi-circular dashed lines.

chirality[28] and Floquet-Hubbard states due to A.C. fields $[29,30]$. Moreover, BICs assisted by MFs enable applications like the storage of qubits[31] and the electrical current switch[32] as well. It should be mentioned that electrons trapped at BICs are prevented to decay into the energy continuum of the environment. Once BICs are undetectable by electrical conductance and accounting for the leakage effect, we benefit of such a remarkable invisibility feature of the BICs. Hence, for the sake of simplicity, we label by cryptography of the MF- 
qubit $\eta_{\uparrow}=\frac{1}{\sqrt{2}}\left(\Psi_{1}+i \Psi_{2}\right)$ when its ZBP signature disappears as a BIC, turning itself undetectable via conductance measurements. As it will be discussed below, we also find an asymmetrical leakage of the MF-qubit. In such a situation, the ZBP is visible in the conductance and we call such a regime by decrypted MF-qubit, since the MF state from the Kitaev chain edge leaks solely into a single QD of the proposed setup (Fig.1). Equivalently, the qubit is recorded over this QD. Our decrypted MF case corresponds to the readout of the qubit in QDs via charge measurement, i.e, the ZB-conductance, as proposed by Flensberg[33]. Otherwise, the encrypted qubit is achieved when the recording is symmetrical over the QDs, but with an invisible ZBP in the conductance in such a way that the readout is off, i.e., the decrypting is not allowed. In this regime, the MF-leaked state at zerobias is split into the QDs, thus becoming BICs. Thereby, we propose that the switch on/off of the readout of the qubit via the ZBP in the QDs consists in a manner of realizing quantum cryptography of the information written in the prepared MFs states $\Psi_{1}$ and $\Psi_{2}$.

The Model.-Below we describe theoretically the setup outlined in Fig.1(a) with a topological Kitaev chain coupled to a double QD setup hybridized with metallic leads[33, 34]. The oversimplified sketch of such a system is depicted in Fig.1(b), which is ruled by the Hamiltonian

$$
\begin{aligned}
\mathcal{H}_{\text {Full }} & =\sum_{\alpha \mathbf{k}} \tilde{\varepsilon}_{\alpha \mathbf{k}} c_{\alpha \mathbf{k}}^{\dagger} c_{\alpha \mathbf{k}}+\sum_{\alpha} \varepsilon_{\alpha} d_{\alpha}^{\dagger} d_{\alpha}+T_{\mathrm{c}}\left(d_{L}^{\dagger} d_{R}+\text { H.c. }\right) \\
& +\mathcal{V} \sum_{\alpha \mathbf{k}}\left(c_{\alpha \mathbf{k}}^{\dagger} d_{\alpha}+\text { H.c. }\right)+\mathcal{H}_{\mathrm{MFs}},
\end{aligned}
$$

where the electrons in the lead $\alpha=L, R$ are described by the operator $c_{\alpha \mathbf{k}}^{\dagger}\left(c_{\alpha \mathbf{k}}\right)$ for the creation (annihilation) of an electron in a quantum state labeled by the wave number $\mathbf{k}$ and energy $\tilde{\varepsilon}_{\alpha \mathbf{k}}=\tilde{\varepsilon}_{\mathbf{k}}-\mu_{\alpha}$, with $\mu_{\alpha}$ as the chemical potential. For the QDs coupled to the leads, $d_{\alpha}^{\dagger}\left(d_{\alpha}\right)$ creates (annihilates) an electron in the state $\varepsilon_{\alpha}$, which is gate tunable. The left-right $\mathrm{QD}$ coupling is $T_{\mathrm{c}}$, while $\mathcal{V}$ stands for the hybridization between these QDs and the leads. Concerning $\mathcal{H}_{\mathrm{MFs}}$ we refer to Ref.[33] which accounts for QDs with large energy spacing of levels between spins up and down due to Zeeman splitting. Consequently, the spinless condition is fulfilled where only spin up state is relevant for the emerging topological superconductivity. In this way, our QDs do not depend on the charging energy as in Refs. $[9,35]$, thus the QDs just couple asymmetrically to the Kitaev chain with complex tunneling amplitudes $\lambda_{L}$ and $\lambda_{R}$, respectively for the left and right QDs as follows

$$
\begin{aligned}
\mathcal{H}_{\mathrm{MFs}} & =i \varepsilon_{M} \Psi_{1} \Psi_{2}+\left|\lambda_{R}\right|\left(e^{i \phi_{R}} d_{R}-e^{-i \phi_{R}} d_{R}^{\dagger}\right) \Psi_{1} \\
& +\left|\lambda_{L}\right|\left(e^{i \phi_{L}} d_{L}-e^{-i \phi_{L}} d_{L}^{\dagger}\right) \Psi_{1},
\end{aligned}
$$

where $\Psi_{1}=\Psi_{1}^{\dagger}$ and $\Psi_{2}=\Psi_{2}^{\dagger}$ account for the MFs lying on the edges of the chain with overlap term $\varepsilon_{M} \sim e^{-L / \xi}$, wherein $L$ and $\xi$ designate respectively, the size of the Kitaev chain and the superconducting coherence length.
We stress that, for a sake of simplicity, by employing the following substitutions $d_{L}=e^{-i \phi_{L}}\left[(\cos \theta) \tilde{d}_{\uparrow}-\right.$ $\left.(\sin \theta) \tilde{d}_{\downarrow}\right], \quad d_{R}=e^{-i \phi_{R}}\left[(\sin \theta) \tilde{d}_{\uparrow}+(\cos \theta) \tilde{d}_{\downarrow}\right]$, $c_{\mathbf{k} L}=e^{-i \phi_{L}}\left[(\cos \theta) \tilde{c}_{\mathbf{k} \uparrow}-(\sin \theta) \tilde{c}_{\mathbf{k} \downarrow}\right]$ and $c_{\mathbf{k} R}=$ $e^{-i \phi_{R}\left[(\sin \theta) \tilde{c}_{\mathbf{k} \uparrow}+(\cos \theta) \tilde{c}_{\mathbf{k} \downarrow}\right]}$ into the Hamiltonian of Eq.(1), in particular at the zero-bias regime ( $\mu_{\alpha}=0 \equiv$ Fermi level of the leads), we obtain

$$
\begin{aligned}
\mathcal{H}_{\mathrm{Full}} & =\sum_{\mathbf{k}, \sigma} \tilde{\varepsilon}_{\mathbf{k}} \tilde{c}_{\mathbf{k} \sigma}^{\dagger} \tilde{c}_{\mathbf{k} \sigma}+\sum_{\sigma} \epsilon_{d \sigma} \tilde{d}_{\sigma}^{\dagger} \tilde{d}_{\sigma}+\mathcal{V} \sum_{\mathbf{k}, \sigma}\left(\tilde{c}_{\mathbf{k} \sigma}^{\dagger} \tilde{d}_{\sigma}+\text { H.c. }\right) \\
& +\mathcal{H}_{\mathrm{MFs}},
\end{aligned}
$$

which mimics an effective single QD coupled to an unique lead both exhibiting an artificial spin degree of freedom $\sigma= \pm 1(\uparrow, \downarrow)$ (see Fig. 1(c) for such a representation).

We call attention that from now on, we label the aforementioned variable by pseudospin. As in Ref.[33], we have topological protection of our findings if the phase difference $\phi_{L}-\phi_{R}=2 n \pi$ is fulfilled, with $n$ integer being tunable via magnetic flux, thus leading to $\cos (2 \theta)=\frac{\Delta \epsilon \cos \left(\phi_{L}-\phi_{R}\right)}{\sqrt{4\left(T_{\mathrm{c}}\right)^{2}+(\Delta \epsilon)^{2}}}, \Delta \epsilon=\varepsilon_{L}-\varepsilon_{R}$ as the detuning of the original spinless QDs, the pseudo-Zeeman gap $\epsilon_{d \uparrow}-\epsilon_{d \downarrow}$, with $\epsilon_{d \sigma}=\frac{\left(\varepsilon_{L}+\varepsilon_{R}\right)}{2}+\frac{\sigma}{2} \sqrt{4\left(T_{\mathrm{c}}\right)^{2}+(\triangle \epsilon)^{2}}$ and

$$
\mathcal{H}_{\mathrm{MFs}}=\varepsilon_{M}\left(\eta_{\uparrow}^{\dagger} \eta_{\uparrow}-\frac{1}{2}\right)+\sum_{\sigma} \bar{\lambda}_{\sigma}\left(\tilde{d}_{\sigma} \eta_{\uparrow}^{\dagger}+\tilde{d}_{\sigma} \eta_{\uparrow}+\text { H.c. }\right),
$$

where we have used $\Psi_{1}=\frac{1}{\sqrt{2}}\left(\eta_{\uparrow}^{\dagger}+\eta_{\uparrow}\right)$ and $\Psi_{2}=$ $\frac{i}{\sqrt{2}}\left(\eta_{\uparrow}^{\dagger}-\eta_{\uparrow}\right)$ in order to build the qubit $\eta_{\uparrow}$ composed by the MFs, namely the MF-qubit, with $\bar{\lambda}_{\uparrow}=\frac{1}{\sqrt{2}}\left(\left|\lambda_{L}\right| \cos \theta+\right.$ $\left.\left|\lambda_{R}\right| \sin \theta\right)$ and $\bar{\lambda}_{\downarrow}=\frac{1}{\sqrt{2}}\left(\left|\lambda_{R}\right| \cos \theta-\left|\lambda_{L}\right| \sin \theta\right)$ as pseudospin-dependent amplitudes. As a result, the pseudoZeeman gap becomes dressed by such an interaction, i.e., $\tilde{\epsilon}_{d \uparrow}-\tilde{\epsilon}_{d \downarrow}$, which will be addressed later on.

We call attention to the system Hamiltonian mapping into Eq.(4), where one can recognize that the device of Fig. 1(b) is equivalent to the QD $\tilde{d}_{\sigma}$ emulating the two original spinless left and right QDs, in particular sidecoupled to $\eta_{\uparrow}$, which corresponds to a QD replacing the Kitaev chain. This opens the possibility of reproducing experimentally the same phenomenon reported here for the topological Kitaev chain by employing QDs, but in the presence of a delocalized Cooper pair split into $\tilde{d}_{\sigma}$ and $\eta_{\uparrow}$ with pairing amplitude $\bar{\lambda}_{\sigma}$ as the terms $\bar{\lambda}_{\sigma}\left(\tilde{d}_{\sigma} \eta_{\uparrow}+\right.$ H.c. $)$ point out. Besides, the normal tunneling between these QDs should be also equal to $\bar{\lambda}_{\sigma}$, i.e., $\bar{\lambda}_{\sigma}\left(\tilde{d}_{\sigma} \eta_{\uparrow}^{\dagger}+\right.$ H.c. $)$, just in order to ensure the emergence of MFs at the so-called "sweet spot" as predicted in Ref.[36] by Flensberg. In such a work, the equivalence of the topological Kitaev chain with a QD system is established by means of an analogous Hamiltonian to our Eq.(4). In this way, this system of QDs hosting MFs becomes an experimental alternative with respect to the topological Kitaev chain. Noteworthy, this QD-like alternative system with MFs was already explored by some of us in Ref.[37] within 
the context of adatoms and STM tips as well as the case of a zero-mode from a regular normal side-coupled QD to a central QD region[38]. For this latter, the qubit $\eta_{\uparrow}$ without the Cooper pairing amplitude (proximity effect) when encrypted would be still protected against the decoherence of the surroundings due to the BIC nature of the state which decouples it from the environment, thus preventing a finite conductance through this channel. Equivalently, BICs do not depend on the proximity effect to occur. However, the decrypted qubit case would not be protected in the same way, once it couples to the environment in contrast to a MF-qubit, which is topologically protected characterized by a pinned ZBP. This characteristic plays the main difference from a regular fermionic zero-mode, wherein the expected ZBP is destroyed by changing external parameters as outlined in Fig.4(a) of Ref.[38] and the readout of the qubit in the central QD region is compromised as a result.

In what follows, we use the Landauer-Büttiker formula for the zero-bias conductance $\mathcal{G}[39]$ to analyze the transport through the QDs, which is

$$
\mathcal{G}=\frac{e^{2}}{h} \int d \varepsilon\left(-\frac{\partial f_{F}}{\partial \varepsilon}\right) \mathcal{T}_{\text {Total }}
$$

where $f_{F}$ stands for the Fermi-Dirac distribution, $\mathcal{T}_{\text {Total }}=\sum_{j} \mathcal{T}_{j j}+\sum_{j} \mathcal{T}_{j \bar{j}}$ encodes the system total transmittance with $j=L, R$ respectively for $\bar{j}=R, L$ to correlate distinct QDs, in which $\mathcal{T}_{j l}=\mathcal{T}_{\uparrow \uparrow}+\mathcal{T}_{\downarrow \downarrow}+\mathcal{T}_{\uparrow \downarrow}+\mathcal{T}_{\downarrow \uparrow}$ dictates the transmittance through the channels $l, j=$ $L, R$ in terms of the coefficients $\mathcal{T}_{\sigma \tilde{\sigma}}$ for the pseudospin representation.

Furthermore, $\mathcal{T}_{j l}=\pi \Gamma \rho_{j l}$ depends upon the Anderson broadening $\Gamma=\pi \mathcal{V}^{2} \sum_{\mathbf{k}} \delta\left(\varepsilon-\tilde{\varepsilon}_{\mathbf{k}}\right)$ [40] and $\rho_{j l}=$ $(-1 / \pi) \operatorname{Im}\left(\tilde{\mathcal{G}}_{d_{j}, d_{l}}\right)$, the densities of states for the spinless QDs from the Hamiltonian of Eq.(1) in terms of the retarded Green's functions $\tilde{\mathcal{G}}_{d_{j}, d_{l}}$, which are given by

$$
\begin{aligned}
\rho_{L L} & =-\frac{1}{\pi} \operatorname{Im}\left\{\cos ^{2} \theta \tilde{\mathcal{G}}_{\tilde{d}_{\uparrow}, \tilde{d}_{\uparrow}}+\sin ^{2} \theta \tilde{\mathcal{G}}_{\tilde{d}_{\downarrow}, \tilde{d}_{\downarrow}}\right. \\
& \left.-\sin \theta \cos \theta\left(\tilde{\mathcal{G}}_{\tilde{d}_{\downarrow}, \tilde{d}_{\uparrow}}+\tilde{\mathcal{G}}_{\tilde{d}_{\uparrow}, \tilde{d}_{\downarrow}}\right)\right\}, \\
\rho_{R R} & =-\frac{1}{\pi} \operatorname{Im}\left\{\sin ^{2} \theta \tilde{\mathcal{G}}_{\tilde{d}_{\uparrow}, \tilde{d}_{\uparrow}}+\cos ^{2} \theta \tilde{\mathcal{G}}_{\tilde{d}_{\downarrow}, \tilde{d}_{\downarrow}}\right. \\
& \left.+\sin \theta \cos \theta\left(\tilde{\mathcal{G}}_{\tilde{d}_{\downarrow}, \tilde{d}_{\uparrow}}+\tilde{\mathcal{G}}_{\tilde{d}_{\uparrow}, \tilde{d}_{\downarrow}}\right)\right\}, \\
\rho_{R L} & =-\frac{1}{\pi} \operatorname{Im}\left\{\sin \theta \cos \theta\left(\tilde{\mathcal{G}}_{\tilde{d}_{\uparrow}, \tilde{d}_{\uparrow}}-\tilde{\mathcal{G}}_{\tilde{d}_{\downarrow}, \tilde{d}_{\downarrow}}\right)\right. \\
& \left.+\cos ^{2} \theta \tilde{\mathcal{G}}_{\tilde{d}_{\downarrow}, \tilde{d}_{\uparrow}}-\sin ^{2} \theta \tilde{\mathcal{G}}_{\tilde{d}_{\uparrow}, \tilde{d}_{\downarrow}}\right\}
\end{aligned}
$$

and

$$
\begin{aligned}
\rho_{L R} & =-\frac{1}{\pi} \operatorname{Im}\left\{\sin \theta \cos \theta\left(\tilde{\mathcal{G}}_{\tilde{d}_{\uparrow}, \tilde{d}_{\uparrow}}-\tilde{\mathcal{G}}_{\tilde{d}_{\downarrow}, \tilde{d}_{\downarrow}}\right)\right. \\
& \left.-\sin ^{2} \theta \tilde{\mathcal{G}}_{\tilde{d}_{\downarrow}, \tilde{d}_{\uparrow}}+\cos ^{2} \theta \tilde{\mathcal{G}}_{\tilde{d}_{\uparrow}, \tilde{d}_{\downarrow}}\right\},
\end{aligned}
$$

here written as functions of the retarded Green's functions $\tilde{\mathcal{G}}_{\tilde{d}_{\sigma}, \tilde{d}_{\tilde{\sigma}}}$ within the mapping on the pseudospin degree. To evaluate $\tilde{\mathcal{G}}_{\tilde{d}_{\sigma}, \tilde{d}_{\tilde{\sigma}}}$, we should employ the equationof-motion method[39] by using Eqs.(3) and (4) as follows: $\varepsilon \tilde{\mathcal{G}}_{\tilde{d}_{\sigma}, \tilde{d}_{\tilde{\sigma}}}=\left[\tilde{d}_{\sigma}, \tilde{d}_{\tilde{\sigma}}^{\dagger}\right]_{+}+\tilde{\mathcal{G}}_{\left[\tilde{d}_{\sigma}, \mathcal{H}_{\text {Full }}\right], \tilde{d}_{\tilde{\sigma}}} \cdot$ As a result, we find the linear system:

$$
\begin{aligned}
& \left(\varepsilon-\epsilon_{d \sigma}-\bar{\lambda}_{\sigma}^{2} K+i \Gamma\right) \tilde{\mathcal{G}}_{\tilde{d}_{\sigma}, \tilde{d}_{\sigma}}-\bar{\lambda}_{\bar{\sigma}} \bar{\lambda}_{\sigma} K \tilde{\mathcal{G}}_{\tilde{d}_{\bar{\sigma}}, \tilde{d}_{\sigma}} \\
& +\bar{\lambda}_{\sigma}^{2} K \tilde{\mathcal{G}}_{\tilde{d}_{\sigma}^{\dagger}, \tilde{d}_{\sigma}}+\bar{\lambda}_{\sigma} \bar{\lambda}_{\bar{\sigma}} K \tilde{\mathcal{G}}_{\tilde{d}_{\bar{\sigma}}^{\dagger}, \tilde{d}_{\sigma}}=1, \\
& -\bar{\lambda}_{\sigma} \bar{\lambda}_{\bar{\sigma}} K \tilde{\mathcal{G}}_{\tilde{d}_{\bar{\sigma}}, \tilde{d}_{\bar{\sigma}}}+\left(\varepsilon-\epsilon_{d \sigma}-\bar{\lambda}_{\sigma}^{2} K+i \Gamma\right) \tilde{\mathcal{G}}_{\tilde{d}_{\sigma}, \tilde{d}_{\bar{\sigma}}} \\
& +\bar{\lambda}_{\sigma} \bar{\lambda}_{\bar{\sigma}} K \tilde{\mathcal{G}}_{\tilde{d}_{\bar{\sigma}}^{\dagger}, \tilde{d}_{\bar{\sigma}}}+\bar{\lambda}_{\sigma}^{2} K \tilde{\mathcal{G}}_{\tilde{d}_{\sigma}^{\dagger}, \tilde{d}_{\bar{\sigma}}}=0, \\
& \bar{\lambda}_{\sigma}^{2} K \tilde{\mathcal{G}}_{\tilde{d}_{\sigma}, \tilde{d}_{\sigma}}+\bar{\lambda}_{\sigma} \bar{\lambda}_{\bar{\sigma}} K \tilde{\mathcal{G}}_{\tilde{d}_{\bar{\sigma}}, \tilde{d}_{\sigma}} \\
& +\left(\varepsilon+\epsilon_{d \sigma}-\bar{\lambda}_{\sigma}^{2} K+i \Gamma\right) \tilde{\mathcal{G}}_{\tilde{d}_{\sigma}^{\dagger}, \tilde{d}_{\sigma}}-\bar{\lambda}_{\sigma} \bar{\lambda}_{\bar{\sigma}} K \tilde{\mathcal{G}}_{\tilde{d}_{\bar{\sigma}}^{\dagger}, \tilde{d}_{\sigma}}=0
\end{aligned}
$$

and

$$
\begin{array}{r}
\bar{\lambda}_{\sigma} \bar{\lambda}_{\bar{\sigma}} K \tilde{\mathcal{G}}_{\tilde{d}_{\bar{\sigma}}, \tilde{d}_{\bar{\sigma}}}+\bar{\lambda}_{\sigma}^{2} K \tilde{\mathcal{G}}_{\tilde{d}_{\sigma}, \tilde{d}_{\bar{\sigma}}}-\bar{\lambda}_{\bar{\sigma}} \bar{\lambda}_{\sigma} K \widetilde{\mathcal{G}}_{\tilde{d}_{\bar{\sigma}}^{\dagger}, \tilde{d}_{\bar{\sigma}}} \\
+\left(\varepsilon+\epsilon_{d \sigma}-\bar{\lambda}_{\sigma}^{2} K+i \Gamma\right) \tilde{\mathcal{G}}_{\tilde{d}_{\sigma}^{\dagger}, \tilde{d}_{\bar{\sigma}}}=0
\end{array}
$$

where $\bar{\sigma}$ is the opposite of $\sigma$ and $K=\left(\varepsilon+\varepsilon_{M}\right)^{-1}+(\varepsilon-$ $\left.\varepsilon_{M}\right)^{-1}$. To perform the analysis of the model in the next section, we make explicit that we have solved the current system numerically.

Results and Discussion.-In the simulations below the temperature $T=0$ is assumed and $\Gamma=40 \mu e V[8,40]$ as the energy scale. The topological Kitaev chain, for a sake of simplicity, is treated as very large, which imposes $\varepsilon_{M} \rightarrow 0$. Thus, in order to make explicit the phenomenon of MF-qubit cryptography, we begin discussing the picture requested for the emergence of such in Fig.2. Fig.2(a) accounts for $\varepsilon_{R}=-2 \Gamma,\left|\lambda_{L}\right|=\left|\lambda_{R}\right|=\lambda=5 \Gamma$, $T_{\mathrm{c}}=1 \Gamma$ and $\varepsilon_{L}=1 \Gamma$, where we verify a ZBP with amplitude of $1 / 4$ in $\mathcal{T}_{\text {Total }}$ of Eq.(5) as a function of $\varepsilon$. This detectable resonance represents the leakage of the MFqubit $\eta_{\uparrow}$ into the double QD setup. Additionally, it also encodes the recording of a decrypted MF-qubit over the left QD, which will be elucidated later on via Figs.3 and 4. On this ground, let us consider the sequence of panels from (b) to (d), which describes the qubit cryptography itself: by changing just $\varepsilon_{L}$, we notice that the ZBP amplitude becomes reduced progressively up to entire quenching in Fig.2(d). In this case, solely a couple of peaks stay visible denoting the dressed pseudo-Zeeman gap $\tilde{\epsilon}_{d \uparrow}-\tilde{\epsilon}_{d \downarrow}$. Indeed, we will clarify that the ZBP becomes BICs, being undetectable by $\mathcal{T}_{\text {Total }}$. It means that if the ZBP is not perceived, we have the accomplishment of the MFqubit cryptography, which appears addressed in detail by Figs. 3 and 4.

Fig.3 exhibits the density plots for $\mathcal{T}_{\text {Total }}, \mathcal{T}_{L L}$ and $\mathcal{T}_{R R}$ spanned by the axis $\varepsilon$ and $\varepsilon_{L}$ for fixed $\varepsilon_{R}=-2 \Gamma$ $\left(\varepsilon_{R}=-1.5 \Gamma\right) \lambda=5 \Gamma\left(\left|\lambda_{L}\right|=1.95 \Gamma\right.$ and $\left.\left|\lambda_{R}\right|=5 \Gamma\right)$ and 


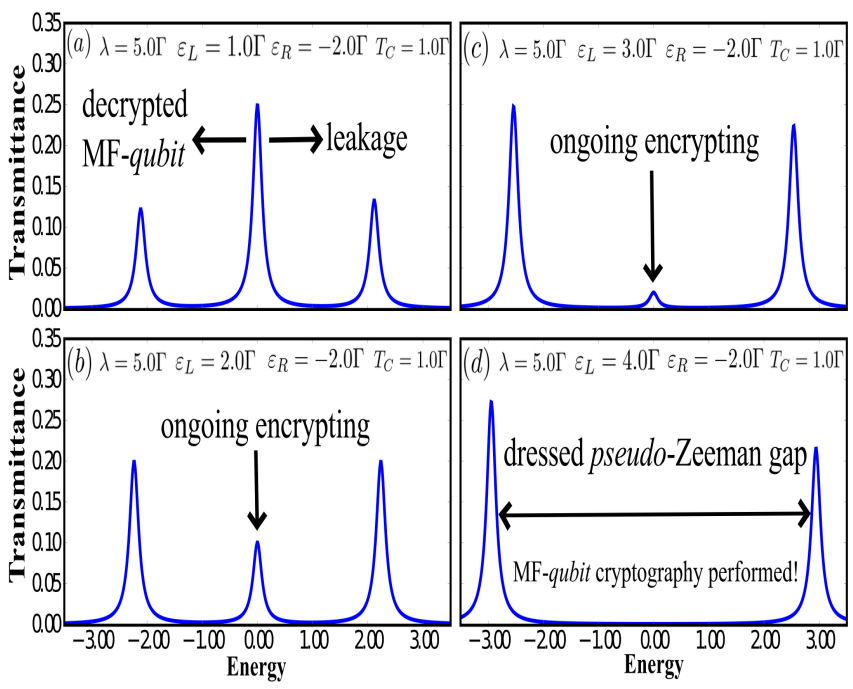

Figure 2. (Color online) $\mathcal{T}_{\text {Total }}$ as a function of $\varepsilon$ : (a) The $\mathrm{ZBP}$ gives the asymmetrical leakage of the MF-qubit $\eta_{\uparrow}$ into the left QD (see also Fig.3). (b)-(c) The increasing of $\varepsilon_{R}$ yields the process for encrypting this qubit, which is characterized by the quenching of the ZBP amplitude. (d) Here the ZBP (the MF-qubit) is hidden as BICs equally split into the QDs, where only the dressed pseudo-Zeeman gap is visible (see also Fig.4).

$T_{\mathrm{c}}=1 \Gamma$. It is worth noticing that all panels in Figs.3(a)(f) present a ZBP structure. However, each one reveals different aspects on the leakage effect. For instance, in Fig.3(a)((d)) we highlight the upper region marked by a yellow dashed ellipse: it gives the domain where the MF-qubit cryptography is allowed, once the ZBP is absent. Figs.3(b)((e)) and (c)((f)) contain the asymmetrical leakage into the $\mathrm{QDs}$ and the decrypted $\mathrm{MF}$-qubit left recording as well. Notice that in the latter, nearby $\varepsilon_{L}=1 \Gamma\left(\varepsilon_{L}=0\right)$, the right QD decouples from the setup, due to $\mathcal{T}_{R R}=0$. This region is then identified by white dashed ellipses in panels (a)-(f) of the same figure. As a result, the MF state is recorded solely at the left QD as Fig.3(b)((e)) ensures. This corresponds to the readout of the qubit by a charge measurement as proposed by Flensberg[33]. Notice that both $\mathcal{T}_{L L}$ and $\mathcal{T}_{R R}$ share the same brightness in their scales, thus pointing out the symmetrical leakage of the MF zero-mode is robust against asymmetrical couplings. Concerning the satellite arcs aside the ZBP in Figs.3(a)-(f), they account for the dressed pseudo-Zeeman gap $\tilde{\epsilon}_{d \uparrow}-\tilde{\epsilon}_{d \downarrow}$. These arcs are predominantly absent, as we can see, at the lower region of Fig.3(a). This points out that BICs away from the ZB limit are also reliable in this device. Thereby, in order to fully understand the underlying physics on the decrypted MF-qubit left recording versus the MF-qubit cryptography, we should consider Fig.4, which uses the same parameters of Fig.2 just for a matter of choice, once for the emergence of the BICs the leakage is always symmetrical even with asymmetrical couplings $\lambda_{L}$ and $\lambda_{R}$ as
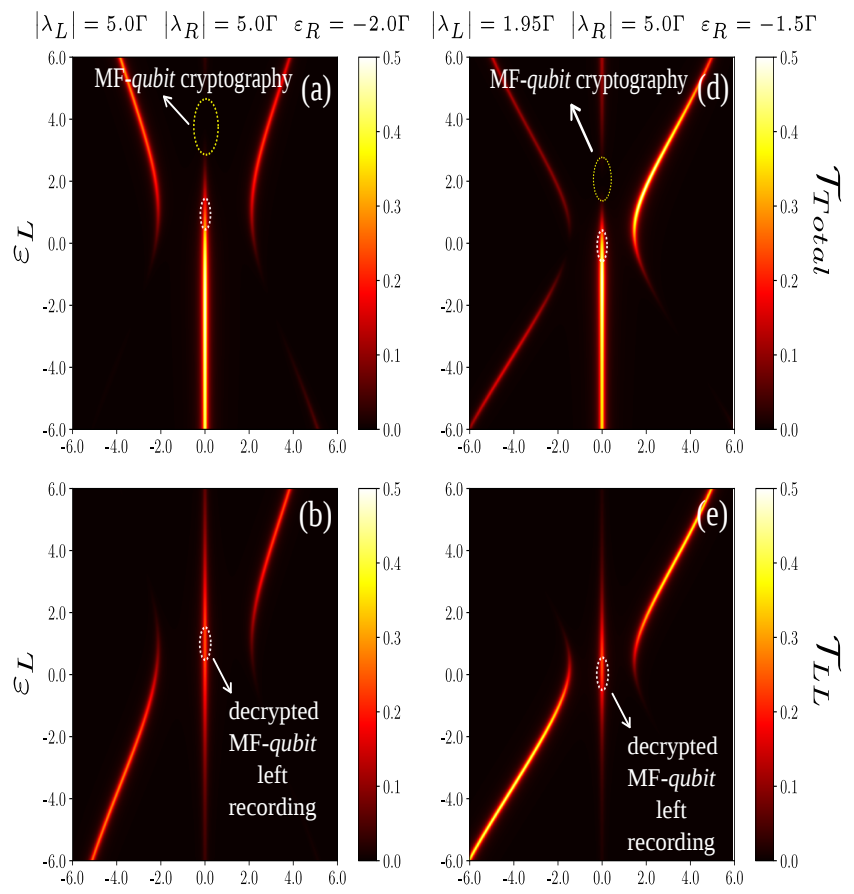

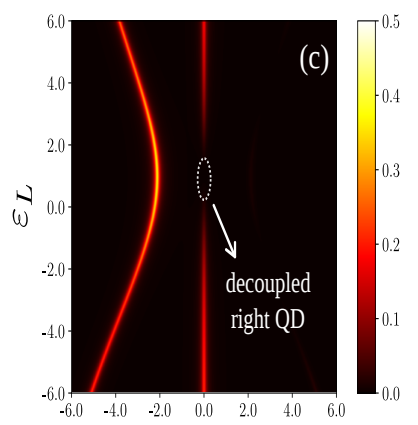

Energy

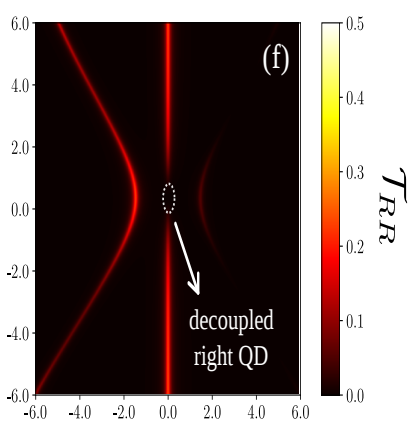

Energy
Figure 3. (Color online) Density plots of: (a) $\mathcal{T}_{\text {Total }}$, (b) $\mathcal{T}_{L L}$ and (c) $\mathcal{T}_{R R}$ spanned by $\varepsilon_{L}$ and $\varepsilon$, with $\varepsilon_{R}=-2 \Gamma$, $\left|\lambda_{L}\right|=\left|\lambda_{R}\right|=\lambda=5 \Gamma$ and $T_{\mathrm{c}}=1 \Gamma$. The ellipses depicted show at zero-bias: i) the region for the MF-qubit cryptography (yellow dashed ellipse) in (a) and ii) the corresponding for the decrypted MF-qubit left recording (white dashed ellipse) in (a) and (b), due to the right QD entirely decoupled from the system as panel (c) shows (white dashed ellipse). Panels (d),(e) and (f) for $\left|\lambda_{L}\right| \neq\left|\lambda_{R}\right|$ give qualitatively the same of (a),(b) and (c) thus ensuring the topological robustness of the results, i.e., the BICs (the encrypted MF-qubit) and the decrypted MF-qubit left recording still occur, but for different set of parameters.

Figs.3(e) and (f) ensure.

In Fig.4(a) the analysis of $\mathcal{T}_{j l}$ shows that the leakage of the MF occurs only over the left QD. In this way, the decrypted MF-qubit situation is achieved: $\mathcal{T}_{L L}$ exhibits a ZBP with amplitude $1 / 4$ in contrast to $\mathcal{T}_{R R}$. Thus in order to understand such an issue, we should focus on the insets. $\mathcal{T}_{R R}$ presents $\mathcal{T}_{\uparrow \uparrow}+\mathcal{T}_{\downarrow \downarrow}$ perfectly phase shifted by $\pi$ with respect to $\mathcal{T}_{\uparrow \downarrow}+\mathcal{T}_{\downarrow \uparrow}$ (Fano dip)[41, 42], thus resulting 

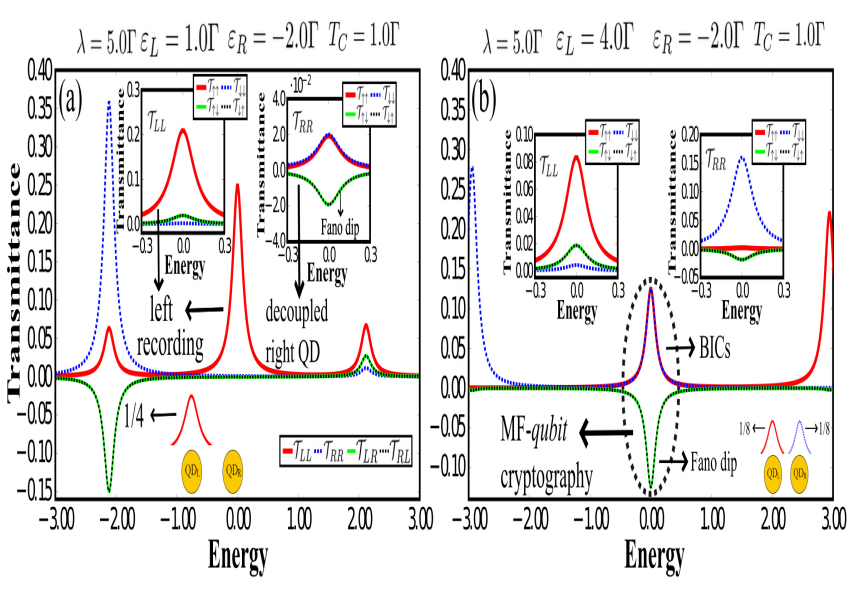

Figure 4. (Color online) $\mathcal{T}_{j l}$ in (a) characterizing the decrypted MF-qubit left recording. $\mathcal{T}_{L L}$ shows a ZBP with amplitude $1 / 4$, while $\mathcal{T}_{R R}$ does not: the inset reveals that $\mathcal{T}_{R R}$ exhibits $\mathcal{T}_{\uparrow \uparrow}+\mathcal{T}_{\downarrow \downarrow}$ perfectly phase shifted by $\pi$ with respect to $\mathcal{T}_{\uparrow \downarrow}+\mathcal{T}_{\downarrow \uparrow}$ (Fano dip). As aftermath, this QD is disconnected from the system. In (b), we have the MF-qubit cryptography: in $\mathcal{T}_{R R}$, the Fano dip is not perfect as before. However, a Fano dip in $\mathcal{T}_{L R}+\mathcal{T}_{R L}$ interferes destructively and exactly with $\mathcal{T}_{L L}+\mathcal{T}_{R R}$. It means that MF-qubit is hidden as BICs equally divided into the QDs.

in a decoupled QD from the setup. For $\mathcal{T}_{L L}, \mathcal{T}_{\uparrow \uparrow}+\mathcal{T}_{\downarrow \downarrow}$ and $\mathcal{T}_{\uparrow \downarrow}+\mathcal{T}_{\downarrow \uparrow}$ interfere constructively. In Fig.4(b) for $\mathcal{T}_{R R}$, the Fano dip in $\mathcal{T}_{\uparrow \downarrow}+\mathcal{T}_{\downarrow \uparrow}$ is not perfect as previously and does not cancel $\mathcal{T}_{\uparrow \uparrow}+\mathcal{T}_{\downarrow \downarrow}$ anymore. Particularly, the Fano dip found in $\mathcal{T}_{L R}+\mathcal{T}_{R L}$ interferes destructively and perfectly with the peak in $\mathcal{T}_{L L}+\mathcal{T}_{R R}$. Finally, this yields the MF-qubit cryptography here proposed. In this way, the recording of the qubit is found secure at two apart sites and hidden as BICs, which are equally split into the QDs and with amplitude 1/8 each. These processes appear outlined in the sketches placed at the lower region of Figs.4(a) and (b).

Interestingly enough, the underlying physics of this cryptography assisted by BICs has a simple picture: the electronic waves traveling forth and back between the QDs $\left(\mathcal{T}_{L R}+\mathcal{T}_{R L}\right)$, in particular at zero-bias, interfere destructively with those waves that only pass through these QDs $\left(\mathcal{T}_{L L}+\mathcal{T}_{R R}\right)$ and as a result, the BICs within the latter emerge. Regarding the satellite arcs aside the ZBP in Figs.4(a) and (b), we should mention that they are also the result of interference processes in $\mathcal{T}_{j l}$ as observed.

Conclusions. - In summary, we have found theoretically that the cryptography of the MF-qubit is feasible in the system of Fig.1(a). We have showed that the recording of the MF-qubit over a single QD is due to an asymmetrical leakage of the MF state into the QDs. The encrypted MF-qubit is performed when the leaking is symmetrical, wherein the MF-leaked state becomes BICs. Thus we reveal a switch on/off mechanism for the readout of the qubit $\eta_{\uparrow}=\frac{1}{\sqrt{2}}\left(\Psi_{1}+i \Psi_{2}\right)$ by means of its ZBP fingerprint on the QDs, which provides a way of performing quantum cryptography regarding the message written inside the MFs states $\Psi_{1}$ and $\Psi_{2}$ initially prepared at the edges of a topological Kitaev chain. Therefore, we trust that our findings can be applied to quantum processing issues in topological quantum computation devices.

Acknowledgments.-This work was supported by CNPq (307573/2015-0), CAPES and São Paulo Research Foundation (FAPESP): grants 2015/26655-9 and 2015/23539-8. We thank L.N. Oliveira for helpful suggestions.
[1] J. Alicea, Rep. Prog. Phys. 75, 076501 (2012).

[2] S. R. Elliott and M. Franz, Rev. Mod. Phys. 87, 137 (2015).

[3] A. Y. Kitaev, Phys. Usp. 44, 131 (2001).

[4] A. A. Zyuzin, D. Rainis, J. Klinovaja, and D. Loss, Phys. Rev. Lett. 111, 056802 (2013).

[5] D. Rainis, J. Klinovaja, L. Trifunovic, and D. Loss, Phys. Rev. B 87, 024515 (2013).

[6] A. Zazunov, P. Sodano, and R. Egger, New J. Phys 15, 035033 (2013).

[7] D. Roy, C. J. Bolech, and N. Shah, Phys. Rev. B 86, 094503 (2012).

[8] E. Vernek, P. H. Penteado, A. C. Seridonio, and J. C. Egues, Phys. Rev. B 89, 165314 (2014).

[9] M. T. Deng, S. Vaitiekenas, E. B. Hansen, J. Danon, M. Leijnse, K. Flensberg, J. Nygard, P. Krogstrup, and C. M. Marcus, Science 354, 6319 (2016).

[10] G. Moore and N. Read, Nucl. Phys. B 360, 362 (1991).

[11] L. Fu, C. L. Kane, and E. J. Mele, Phys. Rev. Lett. 98, 106803 (2007).

[12] L. Fu and C.L. Kane, Phys. Rev. Lett. 100, 096407 (2008).
[13] J. D. Sau, R. M. Lutchyn, S. Tewari, and S. Das Sarma, Phys. Rev. Lett. 104, 040502 (2010).

[14] T. Kawakami and X. Hu, Phys. Rev. Lett. 115, 177001 (2015).

[15] S. N.- Perge, I. K. Drozdov, J. Li, H. Chen, S. Jeon, J. Seo, A. H. MacDonald, B. A. Bernevig, and A. Yazdani, Science 346, 602 (2014).

[16] R. Pawlak, M. Kisiel, J. Klinovaja, T. Meier, S. Kawai, T. Glatzel, D. Loss, and E. Meyer, Nature Partner Journals Quantum Information 2, 16035 (2016).

[17] V. Mourik, K. Zuo, S. M. Frolov, S. R. Plissard, E. P. A. M. Bakkers, and L. P. Kouwenhoven, Science 336, 1003 (2012).

[18] J. von Neumann and E. Wigner, Phys. Z. 30, 465 (1929).

[19] F. H. Stillinger and D. R. Herrick, Phys. Rev. A 11, 446 (1975).

[20] C. W. Hsu, B. Zhen, A. D. Stone, J. D. Joannopoulos, and M. Soljacic, Nature Review Materials, 1, 16048 (2016).

[21] L. H. Guessi, Y. Marques, R. S. Machado, L.S. Ricco, K. Kristinsson, M. S. Figueira, I. A. Shelykh, M. de Souza, and A. C. Seridonio, Phys. Rev. B 92, 245107 (2015). 
[22] L. H. Guessi, R. S. Machado, Y. Marques, L. S. Ricco, K. Kristinsson, M. Yoshida, I.A. Shelykh, M. de Souza, and A. C. Seridonio, Phys. Rev. B 92, 045409 (2015).

[23] W.-J. Gong, X.-Y. Sui, Y. Wang, G.-D. Yu, and X.-H. Chen, Nanoscale Research Letters 8, 330 (2013).

[24] Y. Boretz, G. Ordonez, S. Tanaka, and T. Petrosky, Phys. Rev. A 90, 023853 (2014)

[25] A. Crespi, L. Sansoni, G. D. Valle, A. Ciamei, R. Ramponi, F. Sciarrino, P. Mataloni, S. Longhi, and R. Osellame, Phys. Rev. Lett. 114, 090201 (2015).

[26] C. W. Hsu, B. Zhen, J. Lee, S.-L. Chua, S. G. Johnson, J.D. Joannopoulos, and M. Soljačić, Nature 499, 188 (2013).

[27] Y. Plotnik, O. Peleg, F. Dreisow, M. Heinrich, S. Nolte, A. Szameit, and M. Segev, Phys. Rev. Lett. 107, 183901 (2011).

[28] J. M.-Petit and R. A. Molina, Phys. Rev. B 90, 035434 (2014).

[29] G. D. Valle and S. Longhi, Phys. Rev. B 89, 115118 (2014).

[30] C. González-Santander, P. A. Orellana, and F. Domínguez-Adame, Europhys. Lett. 102, 17012 (2013).

[31] L. S. Ricco, Y. Marques, F. A. Dessotti, R. S. Machado, M. de Souza, and A. C. Seridonio Phys. Rev. B 93,
165116 (2016)

[32] F. A. Dessotti, L. S. Ricco, Y. Marques, L. H. Guessi, M. Yoshida, M. S. Figueira, M. de Souza, Pasquale Sodano, and A. C. Seridonio Phys. Rev. B 94, 125426 (2016).

[33] Karsten Flensberg, Phys. Rev. Lett. 106, 090503 (2011).

[34] D. E. Liu and H. U. Baranger, Phys. Rev. B 84, 201308(R) (2011)

[35] M. Leijnse and K. Flensberg, Phys. Rev. B 84, 140501(R) (2011).

[36] M. Leijnse and K. Flensberg, Phys. Rev. B 86, 134528 (2012).

[37] F.A. Dessotti, L.S. Ricco, Y. Marques, R.S. Machado, L.H. Guessi, M.S. Figueira, M. de Souza, and A.C. Seridonio, Physica E 83, 297 (2016).

[38] F.A. Dessotti, L.S. Ricco, M. de Souza, F.M. Souza, and A.C. Seridonio, J. Appl. Phys. 116, 173701 (2014).

[39] H. Haug and A. P. Jauho, Quantum Kinetics in Transport and Optics of Semiconductors, Springer Series in Solid-State Sciences 123 (Springer, New York, 1996).

[40] P. W. Anderson, Phys. Rev. 124, 41 (1961).

[41] U. Fano, Phys. Rev. 124, 1866 (1961). r d

[42] A. E. Miroshnichenko, S. Flach, and Y. S. Kivshar, Rev. Mod. Phys. 82, 2257 (2010). 